\title{
Effect of a Pharmacist-Driven Monitoring Program and Electronic Health Record on Bleeding Log Completeness and Documentation
}

\author{
Blake Shay, PharmD, MS, BCPS; Julie Kennerly-Shah, PharmD, MHA, BCPS; \\ Marjorie Neidecker, PhD, MEng, RN; Stuart Beatty, PharmD, BCACP, CDE; \\ Leslie Witkoff, RN; Nicole Brown, MS; and Eric Kraut, MD
}

\begin{abstract}
BACKGROUND: Pharmacists have the ability to enhance comprehensive care for bleeding disorders patients by bridging the gap between hemophilia treatment centers (HTC) and specialty pharmacies, specifically by monitoring bleeding logs. In September 2015, a pharmacist-driven monitoring program was implemented through the specialty pharmacy associated with a medical center to improve bleeding log completeness and electronic documentation for HTC patients.
\end{abstract}

OBJECTIVE: To measure the effect of a pharmacist-driven bleeding disorder monitoring program on bleeding log completeness, successful bleeding log documentation in the electronic health record (EHR), and pharmacistdriven clinical interventions using an EHR tool.

METHODS: A single-group pre-post intervention study was conducted of a pharmacist-driven monitoring program. Pre-implementation (January 1, 2014-December 31, 2014), all patients who received and returned a bleeding log following an appointment at the HTC were included; post-implementation (September 1, 2015-December 30, 2015) included patients seen at the HTC who chose to participate in the program for at least 3 months. Before implementation, patient-completed bleeding logs were scanned into the EHR by clinic staff. After implementation, bleeding logs were completed by a pharmacist and documented using a case management tool in the integrated EHR. Bleeding log records successfully documented in the EHR were collected. Completeness was calculated based on 10 clinical data elements for each bleeding log record. Pharmacist-driven interventions resulting from the program in the post-implementation period were recorded.

RESULTS: In the pre-implementation period, 19 of 117 bleeding log records (16.2\%) were documented in the EHR; all $15(100 \%)$ records were documented post-implementation $(P<0.001)$. Among all clinical data elements across all records, 706 of 1,170 data elements were recorded pre-implementation $(60.3 \%)$, and 120 of $150(80.0 \%)$ were recorded post-implementation $(P<0.001)$. Pre-implementation, no logs were $100 \%$ complete; post-implementation, only $6.7 \%$ of logs were fully complete $(P=0.114)$. For the 15 bleeding log records documented in the EHR during the post-implementation period, 14 documented pharmacist-driven clinical interventions occurred. The majority of interventions fell under coordination of care (8 [57.1\%]).

CONCLUSIONS: Improvement in bleeding log completeness and documentation in the EHR was associated with the use of an EHR tool and pharmacistdriven monitoring program.

J Manag Care Spec Pharm. 2018;24(10):1034-39

Copyright $\odot 2018$, Academy of Managed Care Pharmacy. All rights reserved.

\section{What is already known about this subject}

Patients who receive care in hemophilia treatment centers (HTC) have a $40 \%$ reduction in mortality rates and hospitalization rates for bleeding complications from hemophilia.

The 2008 average annual cost per patient for clotting factor therapies was approximately $\$ 155,136$, with an average single hospital admission costing $\$ 76,823$ in 2007.

The National Hemophilia Foundation does not currently promote pharmacists as necessary members of the comprehensive care team.

\section{What this study adds}

As medication experts, pharmacists can be used on the comprehensive care team to identify bleeding events, educate on appropriate dosing regimens, and provide guidance when reviewing patient bleeding logs.

A pharmacist-driven monitoring program for bleeding logs was associated with improved completeness and documentation through the use of an EHR case management tool.

While documentation of patient bleeding events using an integrated case management tool in the EHR can be time consuming, it provides opportunities for patient interventions.

I n the United States, an estimated 20,000 people are living with hemophilia, with hemophilia A affecting 1 in 5,000 male births. ${ }^{1}$ Due to the high cost of clotting factor therapies used to treat or prevent acute bleeding events, the average annual cost per patient in 2008 was approximately $\$ 155,136$, with an average single hospital admission in 2007 costing \$76,823. ${ }^{2-5}$

For preventive treatment, approximately $70 \%$ of people with hemophilia in the United States receive multidisciplinary, comprehensive care in a network of federally funded hemophilia treatment centers (HTCs). ${ }^{1}$ Currently, there are 147 federally funded HTCs in the United States. ${ }^{1}$ The National Hemophilia Foundation recommends that an HTC provide comprehensive care through a team approach that includes physicians, nurses, social workers, physical therapists, and dentists. ${ }^{6}$ Patients who use HTCs will typically use the specialty pharmacy preferred by their insurance payer for dispensing factor products and other bleeding disorder medications. ${ }^{7}$ When patients obtain their medication using this distribution model, the 
comprehensive care team faces several challenges, including ensuring continuity of care, ensuring bleeding log compliance, receiving accurate information related to bleeding events, and ensuring that patients receive their factor products in a timely manner. ${ }^{8,9}$ Despite the high cost of factor products and close coordination with a specialty pharmacy, the National Hemophilia Foundation does not currently promote pharmacists as necessary members of the comprehensive care team.

As opportunities for improvements in comprehensive care exist, the benefits of pharmacists and their potential to maximize care for patients with bleeding disorders should be explored. Studies evaluating treatment adherence and documentation of bleeding episodes in patients with bleeding disorders have demonstrated inadequate data collection of bleeding events due to forgetfulness, time required for treatment, and fluctuation of symptoms. ${ }^{10,11}$ Documentation of bleeding events through bleeding logs is a simple tool that patients and members of the comprehensive care team can use to better understand patient drug use and adherence to therapy. ${ }^{12}$ Accurate, timely, and accessible documentation of patient bleeding events can potentially be used to maximize care by modifying dosing, evaluate patient adherence to treatment regimens, decrease time to therapy when bleeding events occur, and minimize excessive factor product supply for patients who have not had a recent bleed. ${ }^{13,14}$ Pharmacists can provide or assist with these services, which may improve treatment adherence and management of factor products. Therefore, the primary objective of this study was to measure the effect of an integrated electronic health record (EHR) documentation tool used by a pharmacistdriven monitoring program on bleeding log completeness and documentation.

\section{Methods}

This single-group pre-post intervention study took place at a large, academic medical center managing 497 patients at the HTC. In September 2015, a pharmacist-driven monitoring program was implemented to improve bleeding log completeness and electronic documentation for HTC patients. Before program implementation, the HTC requested that patientcompleted bleeding log forms be mailed to the clinic quarterly, where the clinic staff then manually scanned the paper logs into the EHR. Although bleeding logs were completed on a standardized form, no system was in place to ensure completeness. Due to the lag time between bleeding events and mailing logs to the HTC, patient interventions were uncommon, with the exception of severe, emergent bleeds that required emergency care or hospital admission.

\section{Study Setting and Population}

The pharmacist-driven monitoring program was conducted through the specialty pharmacy associated with the medical center. During the pre-implementation period, the patient-completed bleeding log analysis included all patients who received and returned a bleeding log following an appointment at the HTC from January 1, 2014, to December 31, 2014. During the postimplementation period, the pharmacist-completed bleeding log analysis included all patients seen at the HTC from September 1, 2015, to December 30, 2015, who chose to participate in the pharmacist-driven monitoring program for at least 3 months. All hemophilia treatment modalities were included. This study was approved by The Ohio State University Institutional Review Board.

\section{Pharmacist-Driven Monitoring Program}

A pharmacist was placed in the HTC for 4 hours each week to help facilitate patient education and interdisciplinary relationship building with the comprehensive care team. The pharmacist informed patients about an opportunity to participate in the new monitoring program; all interested patients received monthly telephone calls from a pharmacist, regardless of their current pharmacy used for factor dispensing. The pharmacist documented bleeding events using an integrated case management tool in the EHR developed for the monitoring program, which was built in the Epic EHR software (Epic System, Verona, WI) using the Flowsheet documentation function. The tool was designed to include bleeding log data elements that were historically required by the HTC for patient-completed bleeding logs and supported by the National Hemophilia Foundation Steps for Living online education program. ${ }^{15}$

\section{Study Definitions and Outcomes Measures}

A bleed was defined as any bleeding event that required treatment with a factor product or other hemostatic agent. A bleeding log record was generated by the patient for every bleeding event reported since the last recorded bleeding log.

To evaluate the effect of the monitoring and documentation program, 2 categories of outcomes were measured in the pre- and post-implementation periods. First, the percentage of all bleeding log records successfully documented in the EHR within the patient's health record was calculated. Documentation was performed by the HTC clinic staff during the pre-implementation period and by the pharmacist during the post-implementation period. Second, for reported bleeds, the completeness of the bleeding log records was calculated based on 10 clinical data elements on each bleeding log record. Completeness was measured in 3 ways: (1) the percentage of each of the 10 individual data elements completed across all bleeding log records, (2) the percentage of bleeding log records that were $100 \%$ complete, and (3) the percentage of all data elements completed across all bleeding log records.

Two secondary outcomes were collected post-implementation only. Clinical interventions documented through the pharmacy monitoring program were defined by 4 actions (HTC notification, patient education, monitoring, and coordination 


\section{TABLE 1 Baseline Patient Characteristics}

\begin{tabular}{|c|c|c|}
\hline Patient Characteristics & $\begin{array}{l}\text { Pre-Implementation } \\
\text { (Jan.-Dec. 2014) }\end{array}$ & $\begin{array}{l}\text { Post-Implementation } \\
\text { (Sept.-Dec 2015) }\end{array}$ \\
\hline Patients & 27 & 7 \\
\hline Mean age (years) $\pm S D$ & $38 \pm 14.5$ & $31.3 \pm 12.1$ \\
\hline \multicolumn{3}{|l|}{ Bleeding disorder, n (\%) } \\
\hline Hemophilia A, moderate & $(3.7)$ & $1 \quad(14.3)$ \\
\hline Hemophilia A, severe & $2 \quad(7.4)$ & $3 \quad(42.8)$ \\
\hline Hemophilia B, mild & $8 \quad(29.6)$ & $0 \quad(0.0)$ \\
\hline Hemophilia B, moderate & $15 \quad(55.6)$ & $(0.0)$ \\
\hline Hemophilia B, severe & $1 \quad(3.7)$ & $(14.3)$ \\
\hline VWD type I & $(0.0)$ & $(14.3)$ \\
\hline Storage pool & $(0.0)$ & $1 \quad(14.3)$ \\
\hline \multicolumn{3}{|l|}{ Bleeding log record data } \\
\hline $\begin{array}{l}\text { Number of reported bleeding } \\
\text { log records among all patients }\end{array}$ & 117 & 15 \\
\hline
\end{tabular}

of care) that could be used to correct a medication management concern or report a bleeding event to the HTC. The average time required by the specialty pharmacy for telephone encounters was determined by viewing time stamps on telephone encounter documentation in the EHR, which included the time spent collecting bleeding log data and documenting the call.

\section{Data Collection}

Data regarding completeness of bleeding log data elements and documentation of logs in the EHR, as well as type of bleeding disorder, were collected for both study groups through manual review of bleeding log records by a pharmacy resident during the pre- and post-implementation periods. If multiple bleeding events were reported on a single bleeding log, each bleeding event was counted as a unique bleeding log record. Pre-implementation data were collected from paper bleeding logs, and post-implementation data were collected from the EHR. Data elements collected to determine bleeding log completeness included treatment type, type of factor product, year of bleed, month of bleed, day of bleed, location of bleed, administration details, drug lot number, drug expiration, and reason for treatment. To determine bleeding log documentation in the EHR, notes and other media sections were reviewed to determine if paper logs had been scanned or recorded into the EHR. Post-implementation pharmacist-driven clinical interventions and documentation time for telephone encounters were captured through EHR documentation.

\section{Statistical Analysis}

Comparison of pre-post documentation in the EHR and percentage of bleeding logs 100\% complete were made using Fisher's exact test. Comparison of total data elements completed across all bleeding log records pre- versus post-intervention was made using the chi-square test. The level of significance was established a priori as 0.05 . Total pharmacist-driven interventions and documentation time were also reported descriptively. All analyses were conducted using Microsoft Excel (Microsoft, Redmond, WA).

\section{Results}

During the pre-implementation data collection period, 117 bleeding log records were captured, representing 27 patients (mean age [standard deviation, SD] $=38$ [14.5] years, range 22-73 years; Table 1). During the post-implementation period, 15 bleeding log records were captured, representing 7 patients (mean age [SD] $=31.3$ [12.1] years, range 19-52 years). The majority of patients in the pre-implementation group had mild or moderate hemophilia B ( $n=8$ [29.6\%] and $n=15$ [55.6\%], respectively), while severe hemophilia A ( $n=3$ [42.8\%]) was predominate in the post-implementation group. Only 1 patient had bleeding log records recorded in both the pre- and post-implementation periods.

In the pre-implementation period, 19 of 117 bleeding log records (16.2\%) were documented in the EHR, whereas all $15(100 \%)$ records were documented post-implementation $(P<0.001)$. Among all clinical data elements across all records, 706 of 1,170 data elements (60.3\%) were recorded preimplementation, and 120 of $150(80.0 \%)$ were recorded postimplementation $(P<0.001$; Table 2$)$. For pre-implementation completed bleeding log records, 3 of the 10 data elements for completeness were recorded at rates less than $50 \%$. These included medication infusion details (drug expiration, 45.3\% of the observations), reason for treatment (28.2\%), and patient treatment type (0\%). Because patient treatment type was missing in all bleeding log records, no logs were $100 \%$ complete.

For post-implementation completed bleeding log records, 2 of the 10 data elements for completeness were recorded less than $50 \%$ of the time. These data elements included medication infusion details (drug expiration, 26.7\%) and medication infusion details (drug lot number, 6.7\%). Medication infusion details drug lot number was only recorded in 1 of the 15 bleeding log records; therefore, only $6.7 \%$ of logs were fully complete in the post-implementation group, resulting in a nonsignificant improvement $(P=0.114)$.

For the 15 bleeding log records documented in the EHR during the post-implementation period, 14 documented pharmacist-driven clinical interventions occurred. The majority of interventions were coordination of care ( $\mathrm{n}=8$ [57.1\%]; Table 2). Three clinical interventions required the specialty pharmacy to urgently notify the HTC. Time required for documentation of bleeding logs post-implementation showed a mean (SD) of 13.7 (9.1) minutes, with a range of 6-32 minutes. 
TABLE 2 Results for Bleeding Log Record Documentation, Completeness, and Interventions

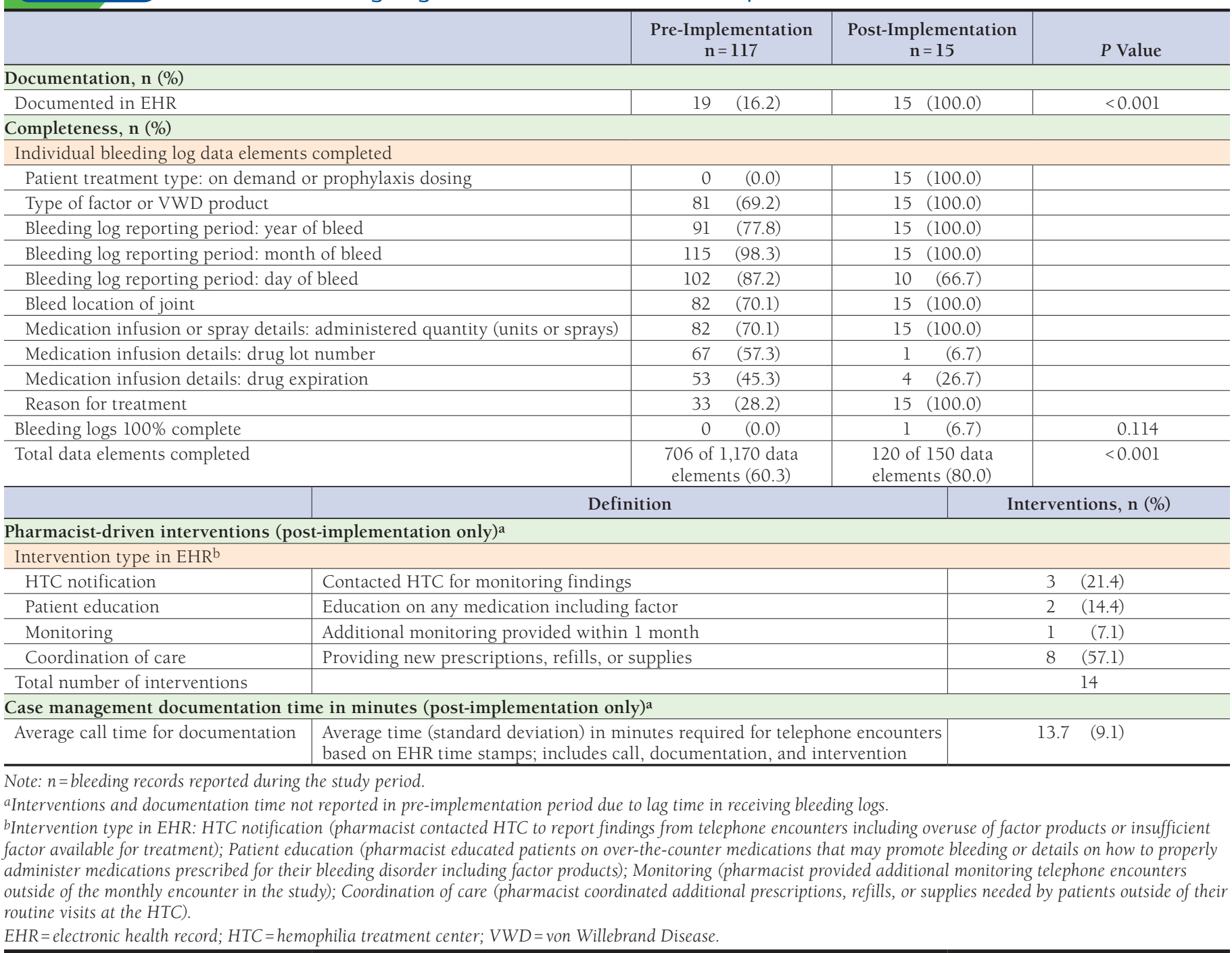

\section{Discussion}

This study demonstrated a positive association between a pharmacist-driven monitoring program using an integrated EHR documentation tool and improved bleeding log completeness and documentation in the EHR among HTC patients with bleeding disorders. Since the pharmacist-driven monitoring program was coupled with EHR use, improvements in bleeding log documentation and completeness should be attributed to both interventions; unfortunately, they could not be evaluated separately for their individual contributions to the results.

While bleeding logs have been the standard of care for determining adherence in patients with bleeding disorders, research has shown that up to $50 \%$ of hemophilia patients do not maintain bleeding logs, and those who do record infusions only adhere to the prescribed frequency $76 \%$ of the time..$^{12,16}$
Due to difficulties in accurately reporting bleeding logs and rapid growth in the area of health information technology, multiple studies have demonstrated the superiority of electronic, patient-reported bleeding logs compared with handwritten documentation. ${ }^{13,14,17-20}$ Walker et al. (2004) showed increased compliance and no change in accuracy when patients used handheld computers versus paper diaries. ${ }^{18}$ Similarly, Collins et al. (2003) demonstrated that an Internet-based electronic patient treatment record improved quality, accuracy, accessibility, and usefulness of patient-generated data in the opinion of patients and health care professionals involved in the study. ${ }^{13}$ Mondorf et al. (2009) successfully demonstrated that a handheld electronic patient diary could serve as a means of treatment optimization and quality assurance in hemophilia care. ${ }^{14}$ 
To our knowledge, the current study is the first to evaluate the use of a health system EHR documentation tool by a pharmacist on bleeding log completeness and documentation. The improvements in bleeding log completeness and documentation in this study suggest utilization of the EHR versus other electronic methods for bleeding logs has the potential to provide benefits that include greater access to information for all health care professionals within a health system and more targeted communication between the HTC and health system specialty pharmacy. Our strategy to use an EHR documentation tool to improve completeness and documentation is not only operationally effective but also has the potential to be clinically effective through documentation of pharmacistdriven clinical interventions.

Based on their training in managing and optimizing complex therapy regimens, pharmacists who specialize in benign hematology can play an integral role in identifying noncompliance patterns, clinical interventions related to prescription and over-the-counter medications, and overuse of factor products that can signify waste or inhibitor formation causing resistance to standard therapies. We observed 3 pharmacist-driven clinical interventions that required notification of the HTC during the post-implementation study period: administration of a 2-fold higher dose than prescribed for on-demand bleeds and 2 bleeding events where available factor doses were not sufficient for treatment. While we recognize that monthly calls by the pharmacist-driven monitoring program may be intrusive to some patients with bleeding disorders, we observed positive anecdotal feedback on bleeding log telephone encounters completed during the study.

\section{Limitations}

This study has several limitations. First, a small sample size of patients was achieved due to the rarity of hemophilia and other bleeding disorder disease states included in the study and the relatively short time window in which the study was conducted. The study compared 2 different time frames from the same HTC, which caused variability in study group sizes and populations.

Second, our results lack generalizability to other HTCs. We are aware that other HTC sites may not be associated with a health system specialty pharmacy or use an electronic form of bleeding log monitoring, which may affect their ability to fully implement a similar program. Additionally, we recognize differences in disease severity, as well as the ratio of bleeding log records to total patients between the pre-post groups. We attribute these differences to having a small study group in the post-implementation period and only being able to select patients seen at the HTC during the post-implementation time frame. While the smaller study population did affect the total number of bleeding log records collected in the post-implementation period, we did not find the difference in severity to have any effect on our findings.
Third, there were no additional pharmacist resources provided for completing telephone encounters during the postimplementation study period. Finally, we realize that the study provides no evaluation or comparison of bleeding log documentation by health care professionals other than pharmacists. While pharmacists may provide the highest level of medication knowledge, we realize there is opportunity to explore the comparison of pharmacists to other health care professionals in managing the monitoring program.

\section{Future Directions}

Following the completion of the study, a full-time pharmacist position was created at the HTC for continuation of the monitoring program. The HTC pharmacist was charged with correlating, documenting, and studying clinical outcomes of the monitoring program and quantifying cost savings for the health system, HTC, and all patients with bleeding disorders. The goal of this position is to develop innovative ways to further increase completeness and documentation of patient-reported bleeding logs. Future studies are warranted to explore the effect of using mobile phone applications and other direct EHR charting methods that can be completed by the patient without requiring telephone encounters with a health care professional. Opportunities for the pharmacist to measure cost reductions associated with clinical interventions may also be possible.

\section{Conclusions}

Improvements in bleeding log completeness and documentation were positively associated with use of an EHR case management tool and pharmacist-driven monitoring program. Implementation of the pharmacist-driven monitoring program incorporated pharmacists into the HTC comprehensive care team and expanded bleeding log monitoring and oversight to patients with bleeding disorders.

\section{Authors}

BLAKE SHAY, PharmD, MS, BCPS, Department of Pharmacy; JULIE KENNERLY-SHAH, PharmD, MHA, BCPS, Department of Pharmacy; LESLIE WITKOFF, RN, Division of Hematology; and ERIC KRAUT, MD, Division of Hematology, Arthur G. James Cancer Hospital \& Richard Solove Research Institute, Columbus, Ohio. MARJORIE NEIDECKER, PhD, MEng, RN, College of Pharmacy and College of Nursing; STUART BEATTY, PharmD, $B C A C P, C D E$, College of Pharmacy; and NICOLE BROWN, MS, College of Medicine, Center for Biostatistics, The Ohio State University, Columbus.

AUTHOR CORRESPONDENCE: Julie Kennerly-Shah, PharmD, MHA, BCPS, Assistant Director of Pharmacy, Arthur G. James Cancer Hospital \& Richard Solove Research Institute, 460 W. 10th Ave., Columbus, $\mathrm{OH}$ 43210-1228. Tel.: 614.655.7357;

E-mail: Julie.Kennerly@osumc.edu. 


\section{DISCLOSURES}

Not outside funding supported this study. The authors have nothing to disclose.

\section{REFERENCES}

1. Centers for Disease Control and Prevention. Hemophilia. Available at: https://www.cdc.gov/ncbddd/hemophilia/index.html. Accessed July 26, 2018.

2. Guh S, Grosse SD, McAlister S, Kessler CM, Soucie JM. Health care expenditures for males with haemophilia and employer-sponsored insurance in the United States, 2008. Haemophilia. 2012;18(2):268-75.

3. Guh S, Grosse SD, McAlister S, Kessler CM, Soucie JM. Health care expenditures for Medicaid-covered males with haemophilia in the United States, 2008. Haemophilia. 2012;18(2):276-83.

4. Chen S. Economic costs of hemophilia and the impact of prophylactic treatment on patient management. Am J Manag Care. 2016;22(5 Suppl):S126-33.

5. Goel R, Krishnamurti L. Mortality, health care utilization and associated diagnoses in hospitalized patients with haemophilia in the United States: first reported nationwide estimates. Haemophilia. 2012;18(5):688-92.

6. National Hemophilia Foundation. Comprehensive medical care: HTCs. Available at: https://www.hemophilia.org/Researchers-Healthcare-Providers/ Comprehensive-Medical-Care-Hemophilia-Treatment-Centers. Accessed July 26, 2018.

7. Blankenship CS. To manage costs of hemophilia, patients need more than clotting factor. Biotechnol Healthc. 2008;5(4):37-40.

8. Lobet S, Hermans C, Lambert C. Optimal management of hemophilic arthropathy and hematomas. J Blood Med. 2014;5:207-18.

9. Escobar MA. Health economics in haemophilia: a review from the clinician's perspective. Haemophilia. 2010;16(Suppl 3):29-34.

10. De Moerloose P, Urbancik W, Van den Berg HM, Richards M. A survey of adherence to haemophilia therapy in six European countries: results and recommendations. Haemophilia. 2008;14(5):931-38.
11. Hacker MR, Geraghty S, Manco-Johnson M. Barriers to compliance with prophylaxis therapy in haemophilia. Haemophilia 2001;7(4):392-96.

12. Du Treil S, Rice J, Leissinger CA. Quantifying adherence to treatment and its relationship to quality of life in a well-characterized haemophilia population. Haemophilia. 2007;13(5):493-501

13. Collins PW, Bolton-Maggs P, Stephenson D, et al. Pilot study of an Internet-based electronic patient treatment record and communication system for haemophilia, Advoy.com. Haemophilia. 2003;9(3):285-91.

14. Mondorf W, Siegmund B, Mahnel R, et al. Haemoassist—a handheld electronic patient diary for haemophilia home care. Haemophilia. 2009;15(2):464-72.

15. National Hemophilia Foundation. Steps for living: treatment logs. Available at: https://stepsforliving.hemophilia.org/basics-of-bleeding-disorders/treatment-basics/treatment-logs. Accessed July 26, 2018.

16. Ho S, Gue D, McIntosh K, Bucevska M, Yang M, Jackson S. An objective method for assessing adherence to prophylaxis in adults with severe haemophilia. Haemophilia. 2014;20(1):39-43

17. Roosendaal G, Drenth ER, Poerschke M, Verkerk EC, Van den Berg HM, Metselaar M. Enhanced communication between care professionals and patients with hemophilia by using a web enabled electronic logbook. Stud Health Technol Inform. 2004;103:191-94.

18. Walker I, Sigouin C, Sek J et al. Comparing hand-held computers and paper diaries for haemophilia home therapy: a randomized trial. Haemophilia. 2004;10(6):698-704.

19. Pattacini C, Rivolta GF, Di Perna C, Riccardi F, Tagliaferri A; Haemophilia Centres Network of Emilia Romagna Region. A web-based clinical record 'x'Emofilia' for outpatients with haemophilia and allied disorders in the Region of Emilia-Romagna: features and pilot use. Haemophilia. 2009;15(1):150-58.

20. Leone JR. Utility of a wireless, handheld monitoring system in the management of hemophilia patients. Comput Inform Nurs. 2011;29(9):521-22. 